\title{
The Sex Doctors
}

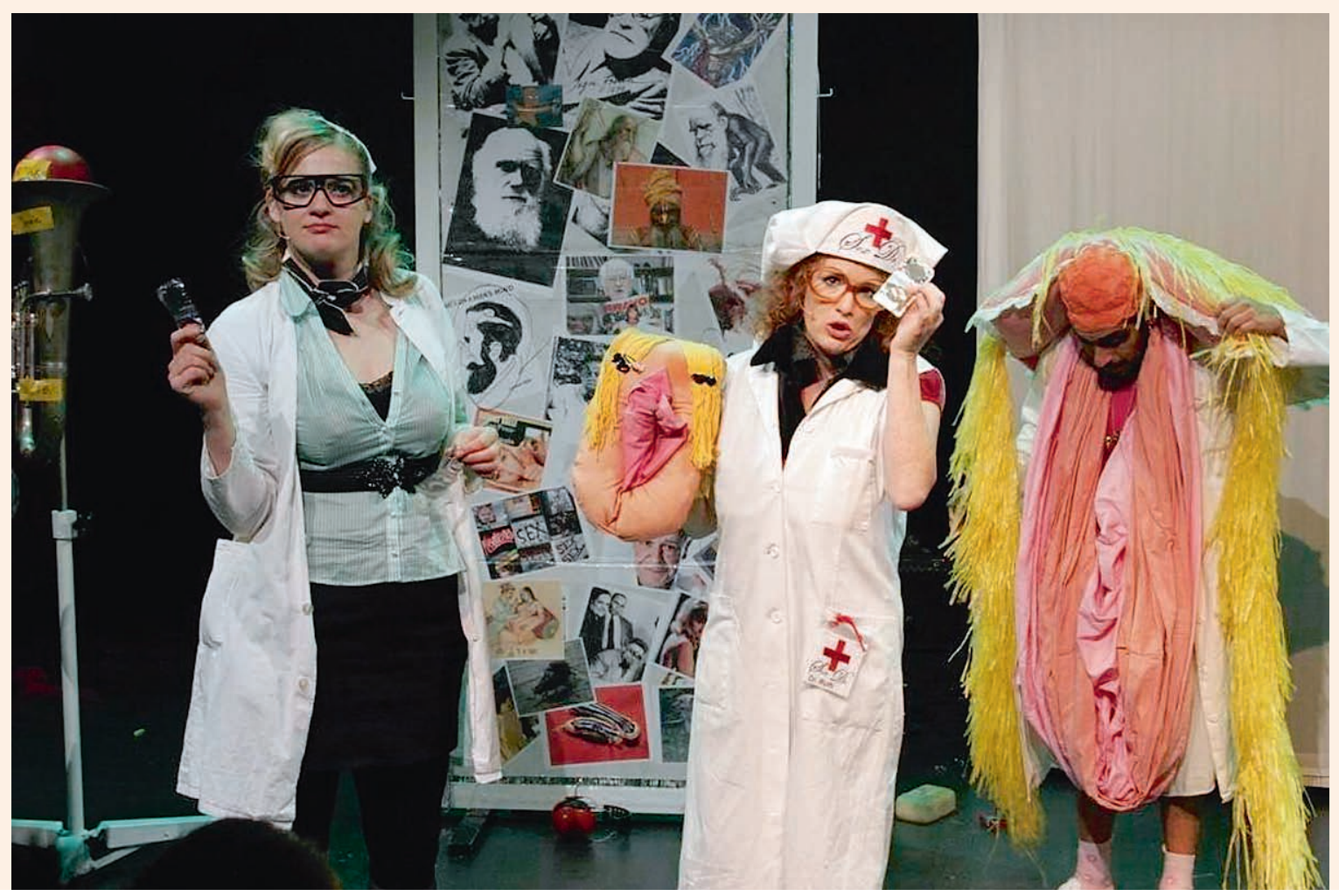

Erhard Taverna

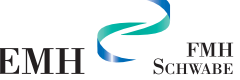

Offiziell wurden wir in der dritten Gymnasialklasse durch den Biologielehrer aufgeklärt. Das geschah in einer Fischzuchtanstalt, wo er reifen Forellen die Eier bzw. den Samen ausquetschte und die Brühe in einem Becken mit Hilfe einer Feder verrührte. Von nun an war uns alles klar.

Die Aufklärungsshow von heute geht andere Wege. «Pussy 'n' Pimmel» für Teens und Oldies ab 12 Jahren mixt schräges Theater mit fetzigem Rock. Die neue Produktion der Zürcher Theatergruppe Kolypan (www.kolypan.ch) kennt keine Tabus. Drei Experten und ihr Assistent führen in wechselnden Rollen durch die Irrgärten der Pubertät, schrill, laut und komisch, aber nie ordinär. Dr. Ruth kämpft gegen Schamgefühle und weiss alles über die Geschichte der Sexualität, von Platons Kugelgestalten über Adam und Eva bis zu den Befragungen des Kollegen Kinsey, furios und präzise vorgetragen, ein atemloser Theaterklamauk mit rasch wechselnden Szenen, begleitet von Gitarren und Schlagzeug. Dr. Helga kennt alle tierischen und menschlichen Begattungsbräuche mit Kamasutra als Zugabe. Dr. Dorian brilliert als Urologe, interpretiert freudianisch feuchte Träume, ist rammelnder Liebhaber und schüchterner Schuljunge. Der
Assistent Eduardo dient allen als Versuchskaninchen, wenn er nicht gerade auf die Pauke haut und die Punkrockband mit dem Publikum im Stroboskoplicht zucken lässt. Die Sex Doctors agieren artistisch und temporeich mit einem bunten Kaleidoskop von Minivorträgen, Sketchen und Songs, handwerklich genau und verblüffend einfallsreich. Erstes Rendezvous, erste Ferienliebe und erste Enttäuschung, Gruppendynamik, Machoallüren und Tussigehabe, Sexphantasien, das erste Mal beim Frauenarzt; alles wird durchgespielt, präzise beobachtet, zum Lachen komisch und dennoch liebevoll. Stets mit Sympathie für unsere schwergeplagte Jugend, die sich in der Regel miserabel vorbereitet in der allgegenwärtigen Pornokultur zurechtfinden muss. Pussy und Pimmel begleiten als geschwätzige Nebendarsteller bauchrednerisch die Show. Zwei kuschelige Plüschfiguren, eindeutig und witzig gemacht, kommentieren und fragen stellvertretend für die teils auch als Schulklassen anwesenden Teens. Ein Ball als Ei auf dem Trichter der Trompete, Tücher und Kissen, ein ausgespannter Strumpf, etwas Ketchup und eine Flasche mit urinfarbiger Limonade, und schon ist die weibliche Anatomie, Menses inklusive, 
perfekt erklärt. Ein Spiel mit Plastiksäcken und Schläuchen, Putzwolle und weiteren Utensilien demonstriert clownesk, aber physiologisch exakt Organaufbau und Funktion beim Mann. Dabei jagt eine Pointe die nächste, es wird durcheinanderdoziert, gestritten, gekreischt und gesungen, immer verständlich und didaktisch hervorragend aufeinander abgestimmt. Romantische Szenen mögen zu kurz kommen, doch es gibt sie. Zum Beispiel beim ersten Zungenkuss, der so aufregend ausfällt, dass die Zungen selbständig weiterzüngeln. Die Kollegen und Kolleginnen leisten während 80 Minuten Schwerarbeit und brauchen dazu auch Wandtafeln und Hellraumprojektoren, noch nie gesehene medizinische Instrumente, Glitzerperücken und jede Menge Phantasie. nen, sie müssen einen Dauerständer haben, und die Mädchen stehen unter Lust- und Stöhnzwang.» Ziel der Show sei es, auch diese falschen Vorstellungen mit Humor zu unterlaufen und zudem älteren Semestern etwas zu bieten, etwa Knutschen zu «Purple Rain» oder brauchbarere Anatomiekenntnisse.

Weitere Kolypan-Projekte waren 2004 ein Erzähltheater «Heidi» zum Mitsingen und Mitfühlen, 2003 eine Lebensberatungsshow «Alergia, Alegría» mit Ritmo Latino und 2001 die «Vladimir Show», das Supermärchen von den verbrauchten und vernachlässigten Spielsachen. Hörspiele kommen dazu und Theaterprojekte wie «Bonjour demain», ein Tanztheater über Migration, oder «King Placebo», eine Reise ins Pharmaland von N. und B. Helbling. Fabienne

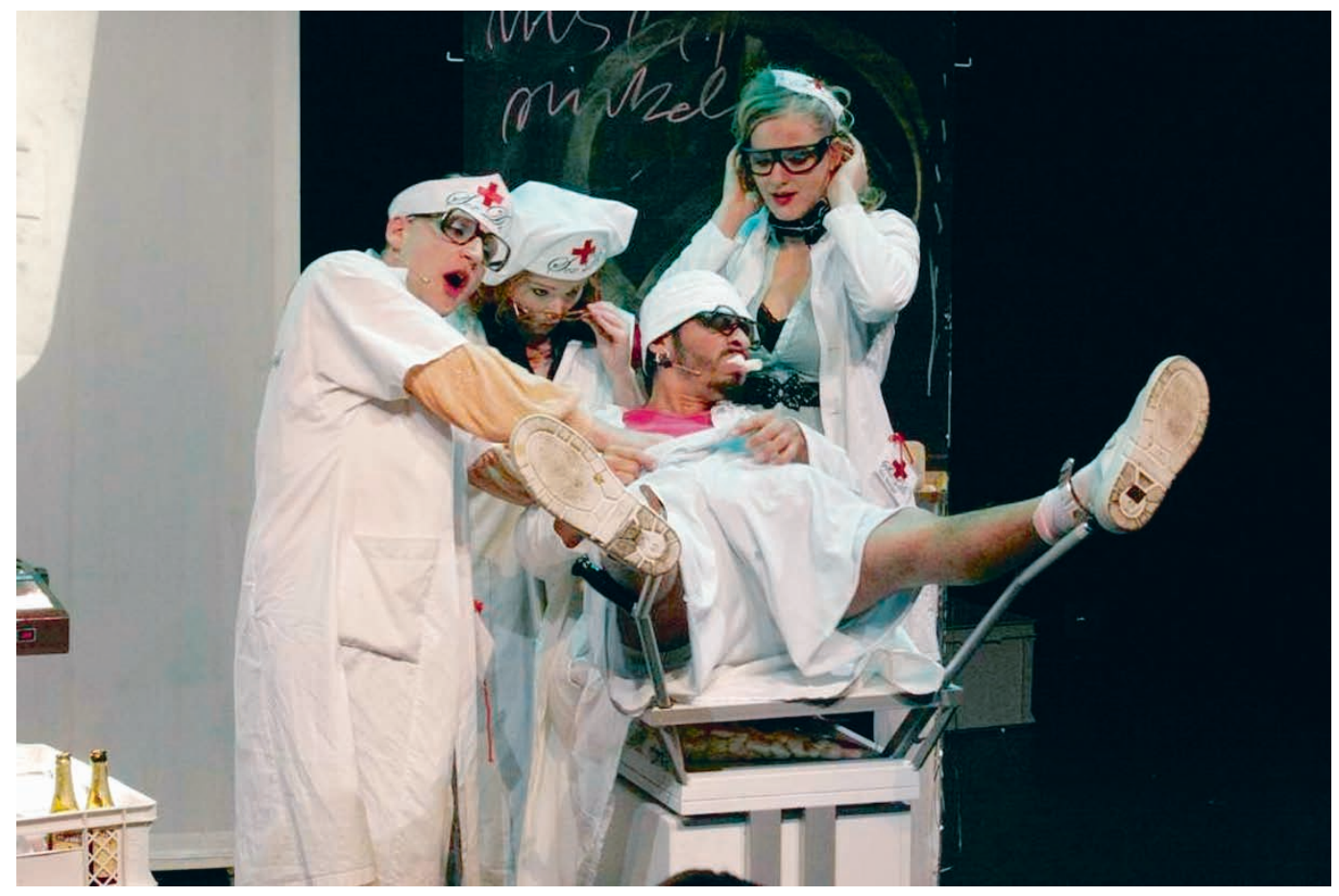

Kolypan wurde im Jahre 2000 von Fabienne Hadorn (geboren 1975) und Gustavo Nañez (geboren 1963) gegründet, um spartenübergreifende Projekte für Kinder und Erwachsene zu realisieren. Mitspieler sind bei «Pussy 'n' Pimmel» Vivien Bullert und Thomas U. Hostettler, Endregie führt Sebastian Nübling. Die Aufklärungsshow wurde mit Fragebogenaktionen in Schulhäusern vorbereitet und ist ausser auf öffentlichen Bühnen auch im Rahmen von Erziehungsprojekten zu erleben. «Die Jungs mei-
Hadorn ist auch von Radio- und TV-Produktionen bekannt, sie arbeitet als Sängerin, Texterin und Tänzerin. Gustavo Nañez lebt als Musiker in Zürich, er komponiert und spielt in verschiedenen Latin-Jazz- und Popformationen.

Spannende Geschichten sind daraus entstanden, von Liebe und Sex für jedes Alter, aufgeführt für die Schule und das grosse Publikum. Wer im schulärztlichen Dienst arbeitet, findet hier eine effektvolle Unterstützung. 